\title{
Estilos de aprendizaje y rendimiento académico en estudiantes de la facultad de Ingeniería de la Universidad Continental, 2011
}

\author{
Learning styles and academic performance in students of the engineering faculty \\ at the Continental University, 2011
}

Bertha Aguilar Bustamante ', Elizabeth Coronel Capacyachi ${ }^{2}$, Walter Goicochea Villavicencio ${ }^{3}$ Universidad Continental

\section{RESUMEN}

Objetivos: Determinar la relación entre estilos de aprendizaje de Bárbara Sóloman (activo-reflexivo, sensorial-intuitivo, visual-verbal, secuencial-global) y el rendimiento académico en estudiantes de la Facultad de Ingeniería de la Universidad Continental de Huancayo. Métodos: Se utilizó el diseño descriptivo correlacional, basado en una muestra no probabilística, con un tamaño muestral de 289 estudiantes de tres Escuelas Académicas Profesionales de Ingeniería, a los cuales se aplicó el Inventario de Estilos de Aprendizaje de Bárbara Sóloman. Resultados: $24,6 \%$ muestra un estado equilibrado del estilo de aprendizaje visual-verbal, el $49,1 \%$ presentan un estado visual moderado; el $20,1 \%$ son visuales muy fuertes; $5,9 \%$ son verbales moderados y sólo el $0,3 \%$ son verbales muy fuertes. Al contrastar la prueba de hipótesis usando la prueba Chi cuadrada y el método de Monte Carlo, cuyo valor es de $p=0,042$; siendo el valor de $p<\alpha$ por lo tanto, se rechaza la hipótesis nula. Conclusiones: En los estudiantes de Ingeniería, existe relación directa y positiva entre el rendimiento académico y el estilo de aprendizaje visual-verbal y no tiene relación con los estilos de aprendizaje activo-reflexivo, sensorial-intuitivo y secuencialglobal. En los estudiantes de la Escuela de Ingeniería Civil se encontró asociación positiva entre rendimiento académico y el estilo de aprendizaje visual-verbal. En la Escuela de Ingeniería de Sistemas e Informática se reporta asociación entre las variables rendimiento académico y el estilo de aprendizaje sensorial-intuitivo; más no se observa relación positiva entre rendimiento académico y el

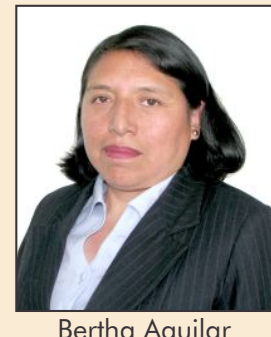

Bertha Aguilar

estilo de aprendizaje activo reflexivo, secuencial-global. En los estudiantes de la Escuela de Ingeniería Ambiental no existe relación entre las variables estudiadas.

Palabras clave: Estilos de aprendizaje, rendimiento académico, estudiantes, ingeniería.

1 Dra. en Educación e Investigación, docente investigadora de la Universidad Continental.

2 Dra. en Educación, docente investigadora de la Universidad Continental.

$3 \mathrm{Mg}$. en Pedagogía, docente investigador de la Universidad Continental. 


\section{ABSTRACT}

Objectives: To determine the relationship between Bárbara Sóloman learning styles (active-reflective, sensory-intuitive, visualverbal, sequential-global) and the academic performance in students of the Engineering faculty at the Continental University from Huancayo. Methods: We used the correlational descriptive design, based on a no-probabilistic sample, with the sample size of 289 students of three professional academic schools of engineering, to which was applied the learning styles inventory by Barbara Sóloman. Results: $24.6 \%$ show a balanced state of the visual - verbal learning style, $49.1 \%$ have a moderated visual state; the $20.1 \%$ are visually very strong; $5.9 \%$ are verbal moderated and only $0.3 \%$ is very strong verbal. By contrasting the hypothesis test using the Chi square test and the Montecarlo method which value of $p=0.042$, where the value $p<\alpha$ therefore the null hyphotesis is rejected. Conclusions: In the engineering students exist a direct and positive relationship between the academic performance and the Visual-Verbal learning style and it's not related whit ActiveReflective, sensory-Intuitive and SequentialGlobal learning styles. In the students in the school of Civil Engineering we found positive association between academic performance and Visual-Verbal learning style. In the school of Systems and Informatic Engineering we reported association between academic performance and the Sensory-Intuitive learning style. But is not observed positive relationship between academic performance and the ActiveReflective, Sequential-Global style. In the students of the school of Environmental Engineering we didn't find any relationship between the studied variables.

Keywords: Learning styles, academic performance, students, engineering.

\section{INTRODUCCIÓN}

Acevedo y Rocha (1) identificaron los estilos de aprendizaje de estudiantes de dos carreras profesionales de la Universidad de Concepción, Chile, provenientes de áreas distintas, Ingeniería Civil Biomédica y Tecnología Médica, para determinar la eventual correlación entre los estilos de aprendizaje y género con el rendimiento académico en el curso de Fisiología Fisiopatología, asignatura de carácter troncal en ambas carreras. Como instrumento de diagnóstico del estilo de aprendizaje se utilizó el Cuestionario Honey - Alonso de Estilos de Aprendizaje (CHAEA) y el rendimiento académico se basó en el registro de las actas de las calificaciones finales obtenidas en la asignatura de FisiologíaFisiopatología. Como resultados se estableció una correlación significativa entre el rendimiento académico y el estilo teórico en los alumnos de Ingeniería Civil Biomédica y con el estilo reflexivo en los alumnos de Tecnología Médica. Al comparar hombres y mujeres, los estudiantes de ambas carreras no muestran diferencias significativas en los estilos de aprendizajes, ni en las calificaciones promedio de la asignatura de Fisiología - Fisiopatología. De los resultados se puede concluir que algunos estilos de aprendizaje pueden eventualmente incidir en el rendimiento académico de los alumnos, pero son independientes del género.

Quintanal (2) elaboró la tesis doctoral cuyo título es "Relación entre los estilos de aprendizaje y el rendimiento académico de la Física y Química de secundaria, defendida en la Universidad Nacional de Educación a Distancia de Madrid - España el día 13 de julio de 2011, dirigida por el Doctor Domingo J. Gallego y que recibió la calificación de Sobresaliente cum laude. El propósito de la investigación fue determinar los estilos de aprendizaje de alumnos de 4 은 de secundaria en cuatro centros concertados, así como las preferencias de dichos estilos, ya sean puros o asociados. Una vez analizados dichos estilos, se procedió a su mejora en uno de los centros, 
empleando una serie de estrategias de enseñanza. Con ello se pretendía mejorar el rendimiento escolar de los alumnos de dicho centro en Física y Química, ya que los estilos de aprendizaje se encuentran vinculados al rendimiento académico. De ahí que se relacionan en las diversas combinaciones de preferencias de los estilos con rendimientos satisfactorios e insatisfactorios. Se arribó a las siguientes conclusiones:

- Se observa la preferencia moderada por todos los estilos de aprendizaje en los centros investigados.

- El estilo educativo de la Institución formativa no influye apreciablemente en los estilos de aprendizaje de sus alumnos.

- Un porcentaje significativo de estudiantes presenta preferencia alta/muy alta hacia diferentes combinaciones de los estilos reflexivo, teórico y pragmático.

- Rendimientos escolares altos aparecen asociados a preferencias alta /muy alta en estilos reflexivo y teórico.

- Rendimientos escolares escasos aparecen asociados a preferencias baja /muy baja en estilos reflexivo y teórico.

- El fortalecimiento de los diversos estilos mediante el empleo de estrategias de enseñanza adecuadas conducen a un incremento del rendimiento escolar.

Amado, et al (3) con el propósito de identificar la relación que existe entre los estilos de aprendizaje y el aprovechamiento académico de estudiantes de nuevo ingreso del Instituto Tecnológico de Mexicali (ITM), México y de la Universidad Pedagógica y Tecnológica de Colombia (UPTC), sede Tunja en el semestre 2008-2. Para conocer los estilos de aprendizaje se aplicó el cuestionario de Richard M. Felder y Bárbara A. Sóloman de la Universidad Estatal de Carolina del Norte. Los resultados indican que la diferencia más notable se observa en los estilos sensorial e intuitivo. Al hacer la comparación, los estudiantes del ITM son $77 \%$ sensoriales y los de la UPTC 27\%, a su vez los estudiantes de la UPTC son $73 \%$ intuitivos y los del ITM 23\%. Al comparar los estilos exclusivamente de los estudiantes de ingeniería de ambas instituciones no se observaron diferencias notables. En ambos grupos son $85 \%$ visuales más que verbales.
Los estudiantes de ingeniería de la UPTC $(91 \%)$ son más visuales que los del ITM (85\%).

Zapata y Flores (4) realizaron una investigación con el objetivo de identificar los estilos de aprendizaje de los alumnos universitarios que se encuentran en el primer año en las diferentes facultades en la Universidad de Piura, a fin de proporcionar a los profesores información acerca de los rasgos característicos de las formas de aprender de sus alumnos. Utilizó el cuestionario producido por Richard Felder y Bárbara Sóloman, organizados en cuatro categorías: activoreflexivo, sensorial-intuitivo, visual-verbal y secuencia-global, las conclusiones fueron: Los alumnos de las Facultades de Ingeniería, Educación y Derecho presentan en su mayoría un estilo reflexivo, seguido del grupo que se ubica en la escala de equilibrado reflexivo-activo. Los alumnos de la Facultad de Comunicación se ubican en su mayoría en el estilo equilibrado reflexivo-activo. Los alumnos de la Facultad de Derecho se orientan principalmente por un estilo sensorial; mientras que los estudiantes de Ingeniería, Educación y Comunicación se encuentran en el nivel equilibrado sensorialintuitivo. Los alumnos de la Facultad de Ingeniería se inclinan hacia un estilo exclusivamente visual, mientras que los alumnos de Educación, Derecho y Comunicación se identifican con los estilos en la escala de equilibrado visual-verbal. Los alumnos de la distintas Facultades de la Universidad de Piura se ubican en un grupo equilibrado secuencial-global. De la misma manera, se identifica a un grupo mayoritario de alumnos de la Facultad de Comunicación que se inclinan por un estilo global.

La Clínica Universitaria de Psicología de la Universidad Continental entre otras actividades, evalúa los estilos de aprendizaje de los estudiantes. Asimismo, se conoce el rendimiento acadé-mico. Sin embargo, se torna necesario investigar la relación que existe entre ambas variables, de modo que los resultados pueden ser utilizados con el objeto de potenciar los estilos de aprendizaje, generar estrategias y estilos de enseñanza por parte de los docentes, a fin 
de mejorar el logro de aprendizajes. De tal manera que el problema de investigación se formula de la siguiente forma: ¿̇ué relación existe entre estilos de aprendizaje y rendimiento académico en los estudiantes de la Facultad de Ingeniería de la Universidad Continental de Huancayo, 2011 ?

En la investigación se consideró la siguiente hipótesis: Existe relación directa y positiva entre estilos de aprendizaje y rendimiento académico de los estudiantes de la Facultad de Ingeniería de la Universidad Continental de Huancayo, 2011.

Se plantea como objetivo general: Determinar la relación que existe entre estilos de aprendizaje y rendimiento académico en estudiantes de la Facultad de Ingeniería de la Universidad Continental de Huancayo 2011. Y como objetivos específicos: Describir las características de los estilos de aprendizaje que presentan los estudiantes de la Facultad de Ingeniería de la Universidad Continental, 2011.

Determinar el nivel de rendimiento académico que presentan los estudiantes de la Facultad de Ingeniería de la Universidad Continental, 2011.

\section{MATERIAL Y METODOS}

El diseño de investigación es descriptivo correlacional más usado en el ámbito de la investigación en psicología. La muestra lo conformaron 289 estudiantes, 194 varones y 95 mujeres de las Escuelas Académicas de Ingeniería Ambiental, Ingeniería Civil, Ingeniería de Sistemas. El método empleado para la selección de la muestra fue de tipo no probabilístico.

Se aplicó el inventario de estilos de aprendizaje producida por Bárbara Sóloman que consta de 28 ítems con dos alternativas "si" o "no". Además se utilizó una hoja de respuestas y el cuadro del perfil de estilos de aprendizaje, que permitió determinar el estilo de aprendizaje en una de las cuatro dimensiones: activo o reflexivo, sensorial o intuitivo, visual o verbal y secuencial o global, en las escalas de Equilibrado, Moderado y Muy Fuerte. La recolección de datos se hizo en administraciones colectivas, por ciclos de estudios, preferentemente se administró en la primera hora de clases, en distintos días, se empleó un tiempo de 15 a 20 minutos.

Respecto a la variable rendimiento académico se utilizó las actas y registros de evaluación de los cuales se obtuvo las notas del promedio ponderado de los estudiantes, según la categorización de rendimiento académico propuesta por Edith Reyes Murillo (5), en el cual establece la valoración de aprendizaje "Deficiente", para notas entre 0 y 10,99; "Bajo" para notas entre 11 y 12,99; "Medio" para notas entre 13 y 14,99; y "Alto" para notas entre 15 y 20.

Se desarrollaron formatos en fichas para facilitar el procesamiento de la información y construir la base de datos.

\section{RESULTADOS}

Para probar la hipótesis general, planteada como: "Existe relación entre estilos de aprendizaje y rendimiento académico en estudiantes de la Facultad de Ingeniería de la Universidad Continental de Huancayo", se utilizó la prueba estadística no paramétrica de Chi Cuadrada debido a que las dos variables han sido categorizadas.

La prueba Chi cuadrada contrasta las siguientes hipótesis:

Ho: Estilos de aprendizaje y rendimiento académico son independientes.

$\mathrm{H1}$ : Estilos de aprendizaje y rendimiento académico son dependientes (existe relación entre las variables)

Para contrastar esta hipótesis se realizó 4 contrastaciones, las cuales consisten en 
cada estilo de aprendizaje con la variable Rendimiento académico categorizado.

Contrastación 1:

Ho: Estilos de aprendizaje Activo-Reflexivo y rendimiento académico son independientes.

$\mathrm{H1}$ : Estilos de aprendizaje Activo-Reflexivo y rendimiento académico son dependientes (existe relación entre las variables)

La Tabla № 01 muestra las variables rendimiento académico categorizado y el estilo de aprendizaje activo-reflexivo donde encontramos que el $55,3 \%$ de los evaluados de la facultad de Ingeniería muestran promedios ponderados calificados como deficientes o bajos, el $37,4 \%$ sus promedios ponderados son calificados como medio y sólo un $7,3 \%$ sus promedios son calificados como altos.

En tanto el 55\% de los evaluados muestra un estado de equilibrio del estilo de aprendizaje Activo-Reflexivo, el $26,3 \%$ son activos moderados; el $13,8 \%$ son reflexivos moderados; sólo un 2,1\% muestran el nivel activo muy fuerte y el $2,8 \%$ son reflexivo muy fuerte.

Al contrastar la prueba de hipótesis usando la prueba Chi cuadrada y la prueba exacta de Fisher (estadístico exacto de Fisher), el cual le da mayor consistencia a la prueba cuyo valor de $p=0,856$; donde el valor de $\mathrm{p}>a$ por lo tanto no se rechaza la hipótesis nula, llegando a la conclusión que el estilo de aprendizaje activo-reflexivo y rendimiento académico son independientes, Tabla № 02.

Tabla Nº1: Distribución de evaluados por rendimiento académico y según estilos de aprendizaje Activo - Reflexivo

\begin{tabular}{|c|c|c|c|c|c|c|c|}
\hline \multirow{2}{*}{\multicolumn{2}{|c|}{$\begin{array}{l}\text { Promedio } \\
\text { Ponderado } \\
\text { Categorizado }\end{array}$}} & \multicolumn{6}{|c|}{ Activo/Reflexivo } \\
\hline & & \multirow{2}{*}{$\frac{\text { Equilibrio }}{27}$} & \multirow{2}{*}{$\begin{array}{c}\begin{array}{c}\text { Reflexivo } \\
\text { moderado }\end{array} \\
5\end{array}$} & \multirow{2}{*}{$\begin{array}{c}\begin{array}{c}\text { Reflexivo } \\
\text { muy fuerte }\end{array} \\
1\end{array}$} & \multirow{2}{*}{$\begin{array}{c}\begin{array}{c}\text { Activo } \\
\text { moderado }\end{array} \\
13\end{array}$} & \multirow{2}{*}{$\begin{array}{c}\begin{array}{c}\text { Activo } \\
\text { muy fuerte }\end{array} \\
0\end{array}$} & \multirow{2}{*}{$\begin{array}{r}\text { Total } \\
46\end{array}$} \\
\hline Deficiente & Evaluados & & & & & & \\
\hline & $\%$ & $9,3 \%$ & $1,7 \%$ & $0,3 \%$ & $4,5 \%$ & $0,0 \%$ & $15,9 \%$ \\
\hline \multirow[t]{2}{*}{ Bajo } & Evaluados & 63 & 14 & 3 & 31 & 3 & 114 \\
\hline & $\%$ & $21,8 \%$ & $4,8 \%$ & $1,0 \%$ & $10,7 \%$ & $1,0 \%$ & $39,4 \%$ \\
\hline \multirow[t]{2}{*}{ Medio } & Evaluados & 60 & 15 & 4 & 27 & 2 & 108 \\
\hline & $\% 1$ & $20,8 \%$ & $5,2 \%$ & $1,4 \%$ & $9,3 \%$ & $0,7 \%$ & $37,4 \%$ \\
\hline \multirow[t]{2}{*}{ Alto } & Evaluados & 9 & 6 & 0 & 5 & 1 & 21 \\
\hline & $\%$ & $3,1 \%$ & $2,1 \%$ & $0,0 \%$ & $1,7 \%$ & $0,3 \%$ & $7,3 \%$ \\
\hline \multirow[t]{2}{*}{ Total } & Evaluados & 159 & 40 & 8 & 76 & 6 & 289 \\
\hline & $\%$ & $55,0 \%$ & $13,8 \%$ & $2,8 \%$ & $26,3 \%$ & $2,1 \%$ & $100,0 \%$ \\
\hline
\end{tabular}

Tabla N 02: Pruebas de Chi-cuadrada

\begin{tabular}{lccccc}
\cline { 2 - 5 } & Valor & GI & $\begin{array}{c}\text { Sig. asintótica } \\
\text { (bilateral) }\end{array}$ & $\begin{array}{c}\text { Sig. exacta } \\
\text { (bilateral) }\end{array}$ & $\begin{array}{c}\text { Sig. Exacta } \\
\text { (unilateral) }\end{array}$ \\
\hline Chi-cuadrada de Pearson & $7,561^{\circ}$ & 12 & $0,818^{{ }^{b}}$ & & \\
Estadístico exacto de Fisher & 6,832 & & & 0,856 \\
N de casos válidos & 289 & & & 0,0 \\
\hline
\end{tabular}




\section{Contrastación 2:}

Ho: Estilos de aprendizaje Sensorial-Intuitivo y rendimiento académico son independientes.

$\mathrm{H} 1$ : Estilos de aprendizaje Sensorial-Intuitivo y rendimiento académico son dependientes (existe relación entre las variables).

En la Tabla № 03 se muestra las variables rendimiento académico categorizado y el estilo de aprendizaje sensorial-Intuitivo, se encuentra que el $49,5 \%$ de los evaluados muestra un estado de equilibrio del estilo de aprendizaje sensorial-Intuitivo; el 31,5\% son sensorial moderados; el 14,2\% son intuitivos moderados; $4,5 \%$ son sensorial muy fuerte $y$ $0,3 \%$ intuitivo muy fuerte.

Al contrastar la prueba de hipótesis usando la prueba Chi cuadrada la prueba exacta de Fisher (estadístico exacto de Fisher), el cual le da mayor consistencia a la prueba cuyo valor de $p=0,067$; siendo el valor de $p>a$ por lo tanto no se rechaza la hipótesis nula, llegando a la conclusión que el estilos de aprendizaje Sensorial-Intuitivo y rendimien to académico son independientes, Tabla № 04.

\section{Contrastación 3:}

Ho: Estilos de aprendizaje Visual-Verbal y rendimiento académico son independientes.

H1: Estilos de aprendizaje Visual-Verbal y rendimiento académico son dependientes (existe relación entre las variables)

La Tabla № 05 se muestra las variables rendimiento académico categorizado y el estilo de aprendizaje visual-verbal. Se observa que el $24,6 \%$ de los evaluados

Tabla N 03: Distribución de evaluados por rendimiento académico y según estilo de aprendizaje Sensorial-Intuitivo

\begin{tabular}{|c|c|c|c|c|c|c|c|}
\hline \multirow{2}{*}{\multicolumn{2}{|c|}{$\begin{array}{l}\text { Promedio } \\
\text { Ponderado } \\
\text { Categorizado }\end{array}$}} & \multicolumn{6}{|c|}{ Sensorial/Intuitivo } \\
\hline & & \multirow{2}{*}{$\frac{\text { Equilibrio }}{17}$} & \multirow{2}{*}{$\begin{array}{c}\begin{array}{c}\text { Intuitivo } \\
\text { moderado }\end{array} \\
15\end{array}$} & \multirow{2}{*}{$\begin{array}{c}\begin{array}{c}\text { Intuitivo } \\
\text { muy fuerte }\end{array} \\
0\end{array}$} & \multirow{2}{*}{$\begin{array}{c}\begin{array}{c}\text { Sensorial } \\
\text { moderado }\end{array} \\
12\end{array}$} & \multirow{2}{*}{$\begin{array}{c}\begin{array}{c}\text { Sensorial } \\
\text { muy fuerte }\end{array} \\
2\end{array}$} & \multirow{2}{*}{$\begin{array}{r}\text { Total } \\
46\end{array}$} \\
\hline Deficiente & Evaluados & & & & & & \\
\hline & $\%$ & $5,9 \%$ & $5,2 \%$ & $0,0 \%$ & $4,2 \%$ & $0,7 \%$ & $15,9 \%$ \\
\hline \multirow[t]{2}{*}{ Bajo } & Evaluados & 58 & 12 & 0 & 40 & 4 & 114 \\
\hline & $\%$ & $20,1 \%$ & $4,2 \%$ & $0,0 \%$ & $13,8 \%$ & $1,4 \%$ & $39,4 \%$ \\
\hline \multirow[t]{2}{*}{ Medio } & Evaluados & 59 & 11 & 1 & 32 & 5 & 108 \\
\hline & $\% 1$ & $20,4 \%$ & $3,8 \%$ & $0,3 \%$ & $11,1 \%$ & $1,7 \%$ & $37,4 \%$ \\
\hline \multirow[t]{2}{*}{ Alto } & Evaluados & 9 & 3 & 0 & 7 & 2 & 21 \\
\hline & $\%$ & $3,1 \%$ & $1,0 \%$ & $0,0 \%$ & $2,4 \%$ & $0,7 \%$ & $7,3 \%$ \\
\hline \multirow[t]{2}{*}{ Total } & Evaluados & 143 & 41 & 1 & 91 & 13 & 289 \\
\hline & $\%$ & $49,5 \%$ & $14,2 \%$ & $0,3 \%$ & $31,5 \%$ & $4,5 \%$ & $100,0 \%$ \\
\hline
\end{tabular}

Tabla N 04: Pruebas de Chi-cuadrada

\begin{tabular}{lrcccc}
\hline & \multicolumn{5}{c}{} \\
\cline { 2 - 5 } & Valor & Gl & $\begin{array}{c}\text { Sig. asintótica } \\
\text { (bilateral) }\end{array}$ & $\begin{array}{c}\text { Sig. exacta } \\
\text { (bilateral) }\end{array}$ & $\begin{array}{c}\text { Sig. Exacta } \\
\text { (unilateral) }\end{array}$ \\
\hline Chi-cuadrada de Pearson & $19,714^{\circ}$ & 12 & $0,073 .^{b}$ & \\
Estadístico exacto de Fisher & 18,694 & & & 0,067 \\
N de casos válidos & 289 & & & \\
\hline
\end{tabular}


Tabla N 05: Distribución de evaluados por rendimiento académico y según estilo de aprendizaje Visual-Verbal

\begin{tabular}{|c|c|c|c|c|c|c|c|}
\hline \multirow{2}{*}{\multicolumn{2}{|c|}{$\begin{array}{l}\text { Promedio } \\
\text { Ponderado } \\
\text { Categorizado }\end{array}$}} & \multicolumn{6}{|c|}{ Visual/Verbal } \\
\hline & & \multirow{2}{*}{$\frac{\text { Equilibrio }}{12}$} & \multirow{2}{*}{$\begin{array}{c}\begin{array}{c}\text { Verbal } \\
\text { moderado }\end{array} \\
2\end{array}$} & \multirow{2}{*}{$\begin{array}{c}\begin{array}{c}\text { Verbal } \\
\text { muy fuerte }\end{array} \\
0\end{array}$} & \multirow{2}{*}{$\begin{array}{c}\begin{array}{c}\text { Visual } \\
\text { moderado }\end{array} \\
27\end{array}$} & \multirow{2}{*}{$\begin{array}{c}\begin{array}{c}\text { Visual } \\
\text { muy fuerte }\end{array} \\
5\end{array}$} & \multirow{2}{*}{$\begin{array}{r}\text { Total } \\
46\end{array}$} \\
\hline Deficiente & Evaluados & & & & & & \\
\hline & $\%$ & $4,2 \%$ & $0,7 \%$ & $0,0 \%$ & $9,3 \%$ & $1,7 \%$ & $15,9 \%$ \\
\hline \multirow[t]{2}{*}{ Bajo } & Evaluados & 26 & 3 & 0 & 61 & 24 & 114 \\
\hline & $\%$ & $9,0 \%$ & $1,0 \%$ & $0,0 \%$ & $21,1 \%$ & $8,3 \%$ & $39,4 \%$ \\
\hline \multirow[t]{2}{*}{ Medio } & Evaluados & 31 & 10 & 1 & 46 & 20 & 108 \\
\hline & $\% 1$ & $10,7 \%$ & $3,5 \%$ & $0,3 \%$ & $15,9 \%$ & $6,9 \%$ & $37,4 \%$ \\
\hline \multirow[t]{2}{*}{ Alto } & Evaluados & 2 & 2 & 0 & 8 & 9 & 21 \\
\hline & $\%$ & $0,7 \%$ & $0,7 \%$ & $0,0 \%$ & $2,8 \%$ & $3,1 \%$ & $7,3 \%$ \\
\hline \multirow[t]{2}{*}{ Total } & Evaluados & 71 & 17 & 1 & 142 & 58 & 289 \\
\hline & $\%$ & $24,6 \%$ & $5,9 \%$ & $0,3 \%$ & $49,1 \%$ & $20,1 \%$ & $100,0 \%$ \\
\hline
\end{tabular}

muestra un estado equilibrado del estilo de aprendizaje visual-verbal; el $49,1 \%$ presentan un estado visual moderado; el $20,1 \%$ son visuales muy fuertes; $5,9 \%$ son verbales moderados y sólo el $0,3 \%$ son verbales muy fuertes.

Al contrastar la prueba de hipótesis usando la prueba Chi cuadrada y el método de Monte Carlo, el cual le da mayor consistencia a la prueba cuyo valor de $p=0,042$; donde el valor de $p<\alpha$ por lo tanto se rechaza la hipótesis nula, llegando a la conclusión que el estilo de aprendizaje visual-verbal y rendimiento académico no son independientes o que si se encuentran relacionados, Tabla № 06 .

\section{Contrastación 4:}

Ho: Estilos de aprendizaje SecuencialGlobal y rendimiento académico son independientes.

$H 1$ : Estilos de aprendizaje SecuencialGlobal y rendimiento académico son dependientes (existe relación entre las variables)

La Tabla № 07, muestra las variables rendimiento académico categorizado y el estilo de aprendizaje secuencial-global donde encontramos que el $50,2 \%$ de los evaluados muestra un estado de equilibrio del estilo de aprendizaje secuencial-global, el $33,9 \%$ son secuenciales moderados; el $12,5 \%$ muestran estilo global moderado; $3,5 \%$ son secuenciales muy fuertes y ningún evaluado muestra el nivel global muy fuerte.

Al contrastar la prueba de hipótesis usando la prueba Chi cuadrada y el método de Monte Carlo, el cual le da mayor consistencia a la prueba cuyo valor de $p=0,140$; donde el valor de $p>a$ por lo tanto no se rechaza la hipótesis nula, llegando a la conclusión que el estilos de aprendizaje secuencial-global y rendimiento académico son independientes, Tabla № 08.

\section{DISCUSIÓN}

El objetivo del estudio fue determinar la relación que existe entre las variables estilos de aprendizaje y rendimiento académico. Referente al rendimiento académico, según la categorización de Edith Reyes Murillo(5), descriptivamente el hallazgo reporta, que el $7,3 \%$ alcanza el nivel académico de alto, el $37,4 \%$ corresponde a estudiantes con niveles de rendimiento medio, el 39,4\% de alumnos clasifican en el nivel de rendimiento académico bajo y el $15,9 \%$ se ubica en la 
Tabla № 06: Pruebas de chi-cuadrado

\begin{tabular}{lccccc}
\hline & \multicolumn{5}{c}{ Sig. de Monte Carlo (bilateral) } \\
\cline { 2 - 6 } & Valor & GI & Sig. & $\begin{array}{c}\text { Límite } \\
\text { Inferior }\end{array}$ & $\begin{array}{c}\text { Límite } \\
\text { Superior }\end{array}$ \\
\hline Chi-cuadrado de Pearson & $19,668^{\mathrm{a}}$ & 12 & $0,088^{\mathrm{b}}$ & 0,082 & 0,093 \\
Estadístico exacto de Fisher & 20,033 & & $0,042^{\mathrm{b}}$ & 0,038 & 0,046 \\
N de casos válidos & 289 & & & \\
\hline
\end{tabular}

Tabla N 07: Distribución de Evaluados por Rendimiento académico y según Estilos de aprendizaje Secuencial-Global

\begin{tabular}{|c|c|c|c|c|c|c|c|}
\hline \multirow{2}{*}{\multicolumn{2}{|c|}{$\begin{array}{l}\text { Promedio } \\
\text { Ponderado } \\
\text { Categorizado }\end{array}$}} & \multicolumn{6}{|c|}{ Secuencial/Global } \\
\hline & & \multirow{2}{*}{$\frac{\text { Equilibrio }}{24}$} & \multirow{2}{*}{$\begin{array}{c}\begin{array}{c}\text { Global } \\
\text { moderado }\end{array} \\
5\end{array}$} & \multirow{2}{*}{$\begin{array}{c}\begin{array}{c}\text { Secuencial } \\
\text { moderado }\end{array} \\
14\end{array}$} & \multirow{2}{*}{$\begin{array}{c}\begin{array}{l}\text { Secuencial } \\
\text { muy fuerte }\end{array} \\
3\end{array}$} & \multirow[t]{2}{*}{$\begin{array}{c}\text { Visual } \\
\text { muy fuerte }\end{array}$} & \multirow{2}{*}{$\begin{array}{r}\text { Total } \\
46\end{array}$} \\
\hline Deficiente & Evaluados & & & & & & \\
\hline & $\%$ & $8,3 \%$ & $1,7 \%$ & $4,8 \%$ & $1,0 \%$ & & $15,9 \%$ \\
\hline \multirow[t]{2}{*}{ Bajo } & Evaluados & 48 & 13 & 48 & 5 & & 114 \\
\hline & $\%$ & $16,6 \%$ & $4,5 \%$ & $16,6 \%$ & $1,7 \%$ & & $39,4 \%$ \\
\hline \multirow[t]{2}{*}{ Medio } & Evaluados & 57 & 17 & 32 & 2 & & 108 \\
\hline & $\% 1$ & $19,7 \%$ & $5,9 \%$ & $11,1 \%$ & ,7\% & & $37,4 \%$ \\
\hline \multirow[t]{2}{*}{ Alto } & Evaluados & 16 & 1 & 4 & 0 & & 21 \\
\hline & $\%$ & $5,5 \%$ &, $3 \%$ & $1,4 \%$ &, $0 \%$ & & $7,3 \%$ \\
\hline \multirow[t]{2}{*}{ Total } & Evaluados & 145 & 36 & 98 & 10 & & 289 \\
\hline & $\%$ & $50,2 \%$ & $12,5 \%$ & $33,9 \%$ & $3,5 \%$ & & $100,0 \%$ \\
\hline
\end{tabular}

Tabla Nº8: Pruebas de chi-cuadrado

\begin{tabular}{lcccccc}
\cline { 2 - 6 } & \multicolumn{7}{c}{ Sig. de Monte Carlo (bilateral) } \\
& Valor & Gl & $\begin{array}{c}\text { Sig. asintótica } \\
\text { (bilateral) }\end{array}$ & Sig. & $\begin{array}{c}\text { Límite } \\
\text { Inferior }\end{array}$ & $\begin{array}{c}\text { Límite } \\
\text { Superior }\end{array}$ \\
\hline $\begin{array}{l}\text { Chi-cuadrado de Pearson } \\
\text { Estadístico exacto de Fisher }\end{array}$ & $14,058^{\mathrm{a}}$ & 9 & 0,120 & $0,114^{\mathrm{b}}$ & 0,108 & 0,120 \\
$\mathrm{~N}$ de casos válidos & 12,675 & & $0,140^{\mathrm{b}}$ & 0,133 & 0,147 \\
\hline
\end{tabular}

escala de rendimiento académico deficiente.

En la investigación se observó, que por más que la Universidad Continental se preocupa por brindar capacitación y actualización constante en temas de evaluación a sus docentes. Sin embargo, se aplican distintos sistemas de calificación hasta cierto punto subjetivas, incluso siendo el sistema vigesimal utilizado, algunos docentes establecen como máxima nota 12.

Adell (6) señala que la nota no siempre es 
indicador fiel de conocimientos conceptuales, procedimentales y actitudinales del estudiante. Refiere que el rendimiento escolar es un producto multicondicionado y multidimensional y que el único responsable no es el centro de formación sino los entornos: familiar, social, cultural y económico.

Respecto a los estilos de aprendizaje en la muestra total de estudiantes destaca la preferencia por el estilo de aprendizaje Visual (93,8\%). Estos resultados coinciden con lo encontrado por Bárbara Sóloman citado por Carter y Lyman (7) en una universidad estadounidense donde en el $80 \%$ de universitarios predomina el estilo visual. Felder y Sóloman (8) respecto a estos resultados concluyen: todo el mundo es a veces visual y verbal. Su preferencia por una u otra categoría puede ser fuerte, moderada o leve. Un equilibrio de los dos es deseable. Del mismo modo, Zapata y Flores(4) hallaron que los alumnos de la Facultad de Ingeniería de la Universidad de Piura se inclinan hacia un estilo exclusivamente visual, mientras que los alumnos de Educación, Derecho y Comunicación se identifican con los estilos en la escala de equilibrado visual-verbal.

Asimismo, Amado, et al (3) aplicando la investigación en situaciones similares encontraron que en la Universidad Pedagógica y Tecnológica de Colombia, sede Tunja y en el Instituto Tecnológico de Mexicali, México en el semestre 2008-2. En ambas instituciones los estudiantes encuestados son $85 \%$ visuales más que verbales. Los estudiantes de ingeniería de la Universidad Pedagógica y Tecnológica de Colombia (91\%) son más visuales que los del Instituto Tecnológico de Mexicali, México que llegan a un (85\%). Ello significa que los participantes aprenden mejor cuando ven fotos, ilustraciones, esquemas, tablas, diagramas, etc. Pues recuerdan mejor lo que ven.

En cuanto a las otras dimensiones se encuentran preferencias equilibradas como Activo-Reflexivo (55\%), Secuencial-Global $(50,2 \%)$, Sensorial-Intuitivo $(49,5 \%)$, Visual-
Verbal (24,6\%). Los estudiantes ActivoReflexivo procesan información aplicando y pensando en la coherencia lógica de la información. Los alumnos Secuencial Global prefieren aprender en secuencia lógica y ordenada para luego tener una visión de la totalidad dando sentido a los detalles, Los estudiantes Sensorial-Intuitivo prefieren resolver problemas siguiendo procedimientos y estrategias que ya conocen, son pacientes con detalles trabajan bien con abstracciones y formulaciones matemáticas. Finalmente, Felder y Sóloman(7) dicen que los estudiantes visuales recuerdan mejor lo que ven: imágenes, diagramas, diagramas de flujo, líneas de tiempo, películas y demostraciones. Alumnos verbales sacan más provecho de las palabras: hablado y escrito, explicaciones. Todo el mundo aprende más cuando se presenta la información tanto visual como verbalmente. Los buenos aprendices son capaces de procesar la información presentada visual o verbalmente.

Estos índices de equilibrios por un lado podría interpretarse como adecuados en la medida que los estilos no son fijos. Revilla (1998) citado por Jesús (9) al respecto asevera que se ajustan al docente, al tipo de asignatura o al contexto estará reflejando que los alumnos tienen recursos para adaptarse a las exigencias y modalidades enseñanza-aprendizajes pero por otro lado, pueden entenderse como indicador de indecisión toda vez que cuando no se tiene definidos nuestros estilos y al ser empleados inadecuadamente estas pueden obstaculizar el buen desempeño académico, ya que se espera que un futuro ingeniero debe aplicar unos estilos más que otros, resaltando que ningún estilo es bueno o malo.

A partir de las 4 contrastaciones, se concluye que en los estudiantes de Ingeniería de la Universidad Continental, existe relación directa y positiva entre el rendimiento académico y el estilo de aprendizaje VisualVerbal y no tiene relación con los estilos de aprendizaje Activo-Reflexivo, SensorialIntuitivo y Secuencial-Global. 


\section{REFERENCIAS BIBLIOGRÁFICAS}

1. Acevedo C, Rocha F. Estilos de Aprendizaje, género y rendimiento Académico. Estilos de Aprendizaje. $2011 ; 8(8): 71-84$.

2. Quintanal F. Incidencia de los estilos de aprendizaje en el rendimiento académico de la física y química de secundaria. Revista Estilos de Aprendizaje. 2011 ; 8(8): 198 -223.

3. Amado M, Brito R, García A, Guerrero $M$, Cuervo C. Estilos de aprendizaje de estudiantes del Instituto Tecnológico de Mexicali, México y la Universidad Pedagógica y Tecnológica de Colombia [on line] 2008.

4. Zapata M, Flores L. Identificación de los estilos de aprendizaje en estudiantes universitarios. Revista Estilos de Aprendizaje. 2008; 2(2): 2-24.

5. Reyes E. Influencia de programa curricular y del trabajo docente en el aprovechamiento escolar en historia del Perú de alumnos del 3er grado de educación secundaria. [Tesis para optar el grado académico de Doctora en Educación]. Lima: Universidad Nacional Mayor de San Marcos; 1988.

6. Adell A. Estrategias para mejorar el rendimiento académico de los adolescentes. Madrid: Pirámide. 2002.

7. Carter C. Lyman S. Orientación vocacional. México: Prentice-Hall; 1997.

8. Felder R, Soloman B. (n.d). Learning Styles and Strategies. [Recuperado el 8 de abril del 2012]. En: http://www.4ncsu.edu/unity/lockers/ users/f/felder/public/ILS dir/styles. htm.

9. Jesús M. Inteligencia, rendimiento académico y estilos de aprendizaje en estudiantes de medicina humana de la Universidad Nacional del Centro del Perú. [Tesis para optar el grado de Magister en Psicología]. Lima: Universidad de San Martín de Porres; 2004.
Correo electrónico:

berycorita14@hotmail.com jcoronel@continental.edu.pe wgoicochea@continental.edu.pe 